Open Access

\title{
Histone methylation levels correlate with TGFBIp and extracellular matrix gene expression in normal and granular corneal dystrophy type 2 corneal fibroblasts
}

Yong-Sun Maeng ${ }^{1}$, Ga-Hyun Lee ${ }^{1}$, Seung-II Choi ${ }^{1}$ Kyu Seo Kim² and Eung Kweon Kim ${ }^{1,3^{*}}$

\begin{abstract}
Background: TGF $\beta 1$-induced expression of transforming growth factor $\beta$-induced protein (TGFBlp) and extracellular matrix (ECM) genes plays a major role in the development of granular corneal dystrophy type 2 (GCD2: also called Avellino corneal dystrophy). Although some key transcription factors are known, the epigenetic mechanisms modulating TGFBIp and ECM expression remain unclear. We examined the role of chromatin markers such as histone $\mathrm{H} 3$ lysine methylation (H3Kme) in TGF 31 -induced TGFBlp and ECM gene expression in normal and GCD2derived human corneal fibroblasts.
\end{abstract}

Methods: Wild-type $(n=3)$, GCD2-heterozygous $(n=1)$, and GCD2-homozygous $(n=3)$ primary human corneal fibroblasts were harvested from human donors and patients prepared. Microarray and gene-expression profiling, Chromatin immunoprecipitation microarray analysis, and Methylated DNA isolation assay-assisted CpG microarrays was performed in Wild-type and GCD2-homozygous human cells.

Results: Transcription and extracellular-secretion levels of TGFBlp were high in normal cells compared with those in GCD2-derived cells and were related to H3K4me3 levels but not to DNA methylation over the TGFBl locus. TGF $\beta 1$ increased the expression of TGFBIp and the ECM-associated genes connective tissue growth factor, collagen-a2[I], and plasminogen activator inhibitor-1 in normal corneal fibroblasts. Increased levels of gene-activating markers ( $\mathrm{H} 3 \mathrm{~K} 4 \mathrm{me} 1 / 3$ ) and decreased levels of repressive markers (H3K27me3) at the promoters of those gene accompanied the changes in expression. TGF $\beta 1$ also increased recruitment of the H3K4 methyltransferase MLL1 and of SET7/9 and also the binding of Smad3 to the promoters. Knockdown of both MLL1 and SET7/9 significantly blocked the TGF $\beta 1$-induced gene expression and inhibited TGF $\beta 1$-induced changes in promoter H3K4me1/3 levels. Those effects were very weak, however, in GCD2-derived corneal fibroblasts.

Conclusions: Taken together, the results show the functional role of H3K4me in TGF 1 1-mediated TGFBIp and ECM gene expression in corneal fibroblasts. Pharmacologic and other therapies that regulate these modifications could have potential cornea-protective effects for granular corneal dystrophy.

Keywords: GCD2, TGFBIp, Extracellular matrix, Histone methylation

\footnotetext{
* Correspondence: mysdm70@gmail.com

'Department of Ophthalmology, Corneal Dystrophy Research Institute,

Yonsei University College of Medicine, 250 Seongsanno, Seodaemun-gu, Seoul 120-752, South Korea

${ }^{3}$ Institute of Vision Research, Severance Biomedical Science Institute, Brain

Korea 21 Plus Project for Medical Science, Yonsei University College of

Medicine, Seoul, South Korea

Full list of author information is available at the end of the article
} 


\section{Background}

Granular corneal dystrophy type 2 (GCD2, also called Avellino corneal dystrophy) is an autosomal dominant disorder caused by an arginine-to-histidine substitution at codon $124(\mathrm{R} 124 \mathrm{H})$ of the transforming growth factor $\beta$-induced gene (TGFBI) on chromosome 5q31.8. TGFBI encodes a protein of 683 amino acids (TGFBIp) [1, 2]. TGFBIp is an extracellular matrix (ECM) protein that is structurally homologous to the axon-guidance protein fasciclin I [3-5]. TGFBIp contains four tandem repeats of fasciclin I domains and an EMI protein-protein interaction domain and plays a key role in a variety of cellular responses including adhesion, migration, proliferation, angiogenesis, and wound healing [6-8]. The age-dependent, progressive accumulation of hyaline and amyloid-containing TGFBIp in the corneal stroma is a hallmark of GCD2, which is characterized by the production of TGFBIp in the corneal epithelia and stroma, interfering with corneal transparency [9]. Moreover, homozygous $\mathrm{R} 124 \mathrm{H}$ mutations in TGFBI cause the severe phenotype of GCD2 characterized by early onset and confluent superficial opacity $[10,11]$. The molecular mechanisms and cellular role of TGFBIp in corneal dystrophy pathogenesis are poorly understood.

TGF $\beta 1$ induces the progressive accumulation of TGFBIp and ECM proteins such as collagens, fibronectin, and keratin in the corneal epithelia and stroma [12-14]. TGF $\beta 1$ increases ECM accumulation through the induction of its downstream effector, connective tissue growth factor (CTGF) [15, 16], and by decreasing matrix degradation through the inhibition of proteases or the activation of protease inhibitors such as plasminogen activator inhibitor-1 (PAI-1) in glomerular mesangial cells [17]. TGF $\beta 1$ can regulate TGFBIp expression through Smad transcription factors and E-box-dependent mechanisms [18-22]. However, the subtle nuclear-chromatin mechanisms involved in the TGF- $\beta 1$-induced expression of key ECM genes in corneal fibroblasts are not clear.

Gene regulation by extracellular stimuli involves not only transcription factors binding to their cognate DNA binding sites but also epigenetic changes in chromatin without alterations in DNA sequence. Post-translational modifications, including acetylation, methylation, and ubiquitination at key lysines, on the amino-terminal tails of nucleosomal histones such as $\mathrm{H} 3$ and $\mathrm{H} 4$ play a key role in modulating chromatin structure and gene transcription [23, 24]. They form a "histone code" that can dictate the transcriptional outcomes of gene activation or repression [25]. In general, the acetylation of H3 lysines (H3KAc) is associated with active gene transcription, whereas the methylation of $\mathrm{H} 3$ lysines (H3Kme) can be associated with either active or inactive gene promoters, depending on the position of the modified lysine. H3KAc is mediated by histone acetyl transferases, whereas $\mathrm{H} 3 \mathrm{Kme}$ is mediated by histone methyltransferases (HMTs). HMTs can mono-methylate, di-methylate, or tri-methylate (H3Kme1, H3Kme2, or $\mathrm{H} 3 \mathrm{Kme}$, respectively) specific lysine residues, thereby adding another epigenetic regulatory layer [24].

The methylation of the fourth lysine in H3 (H3K4me) is usually associated with gene activation and transcriptional elongation and is mediated by HMTs such as SET1, MLL1-4, and SET7/9 [24, 26-28]. H3K9me, on the other hand, is generally associated with gene repression and is mediated by HMTs such as SUV39H1, G9a, and SETDB1/ESET [26]. Other H3 lysines, including H3K27, H3K36, and H3K79, can also be methylated to various degrees [26]. In addition, the discovery of histone lysine demethylases has added another dimension to gene regulation [29]. Together, these factors create a fine balance of gene regulation, a disruption of which could result in abnormal gene expression and disease phenotypes.

It is not known whether lysine methylation in the $\mathrm{H} 3$ promoter plays a role in the TGF $\beta 1$-induced transcription of TGFBIp and ECM-associated genes in corneal fibroblasts. Here, we show that the transcription and ECM secretion levels of TGFBIp are high in normal cells compared with those in GCD2 cells and are related to H3K4me3 levels. We also show that TGF 31 leads to the enrichment of H3K $4 \mathrm{me} 1 / 3$ and the depletion of H3K27me3 markers on the promoters of TGFBIp and ECM-associated genes in corneal fibroblasts. Furthermore, H3K4, HMT, MLL1, and SET7/9 all seemed to play a role in the TGF $\beta 1$-induced expression of TGFBIp and ECM genes. These data show novel epigenetic mechanisms of TGF $\beta 1$ actions in corneal fibroblasts related to TGFBIp and ECM deposition and GCD2 pathogenesis.

\section{Methods \\ Materials}

Recombinant TGF $\beta 1$ was obtained from R\&D Systems (Minneapolis, $\mathrm{MN}$ ); normal rabbit IgG, normal mouse IgG, and ChIP-Grade Protein A/G Magnetic Beads were obtained from Thermo Scientific, (Rockford, IL, USA). The following antibodies were used in western-blot analyses and ChIP assays: anti-smad3 (ab28379), anti-SET7/ 9 (ab13731), anti-histone H3 (ab100938), anti-histone H3 trimethyl K9 (ab8898), and anti-H3 trimethyl K27 (ab1782) from Abcam (Cambridge, MA); anti-MLL1 (05-765), anti-H3 monomethyl K4 (07-436), and antiH3 trimethyl K4 (07-473) from Millipore (Millipore Corp., Bedford, MA); anti-TGFBIp (AF2935) from R\&D Systems; and anti- $\beta$-actin (A-5441) from Sigma-Aldrich (St. Louis, MO). MLL1 shRNA lentivirus (sc-38039-v), SET7/9 shRNA lentivirus (sc-44094-v), and control shRNA lentivirus (sc-108080) was purchased from Santa Cruz Biotechnology Inc. (Heidelberg, Germany). 
Isolation and culture of primary corneal fibroblasts

Wild-type $(n=2)$ and GCD2-homozygous $(n=2)$ (Arg124His mutation) primary human corneal fibroblasts were harvested from human donors (age matched) and patients prepared using a previously published method [30]. Donor confidentiality was maintained according to the Declaration of Helsinki, and the Severance Hospital IRB Committee (CR04124), Yonsei University approved the protocol. The wild-type and GCD2 cells were diagnosed by DNA sequencing of TGFBI mutations. After the removal of the corneal buttons from the donors for penetrating keratoplasty, the remaining corneal rims were harvested for culture of the corneal fibroblasts. The medical records of the donors from the eye bank of Yonsei University Severance Hospital did not show any genetic or systemic metabolic disease. The fibroblasts grown from the pieces of corneal rims were treated as healthy controls. The corneal fibroblast cells were cultured in DMEM supplemented with $10 \%$ fetal bovine serum at $37{ }^{\circ} \mathrm{C}$ in a humidified incubator with $95 \%$ air and $5 \%$ CO2.

\section{Microarray and gene-expression profiling}

Transcriptional profiles were evaluated in two independent cell preparations using a cDNA microarray (GeneChip Human Gene 1.0 ST Array, GeneChip; Affymetrix, Santa Clara, CA) containing approximately 29,000 genes. To determine the variation and average changes ( $x$-fold) in the expression of different genes in each sample and to compare the samples, we analyzed four samples (normal sample pairs 1 and 2 (age matched) and GCD2-homozygous (Arg124His mutation) sample pairs 1 and 2) in an additional microarray analysis without total RNA pooling. Total RNA was extracted from the cells (TRIzol; Invitrogen, Carlsbad, CA), followed by purification (RNeasy kit; Qiagen, Valencia, CA) to remove residual DNA. The concentration of total RNA was determined by UV spectrophotometry (ND-1000 UV-vis Spectrophotometer; Nanodrop Technologies, Wilmington, DE). Two quality controls were used for each RNA sample: (1) an $\mathrm{A}_{260} / \mathrm{A}_{280}$ ratio between 1.7 and 2.3 and (2) an electropherogram showing two distinct ribosomal peaks corresponding to $18 \mathrm{~S}$ and $28 \mathrm{~S}$ RNAs, respectively, at a ratio of $28 \mathrm{~S} / 18 \mathrm{~S}>0.5$ with minimal or no degradation.

$100 \mathrm{ng}$ of total RNA was used for the microarray experiment. GeneChip Human Gene 1.0 ST Arrays (Affymetrix, Santa Clara, USA) were used. Whole microarray experiment was performed according to the manufacturers protocol. The Affymetrix GeneChip Whole Transcript (WT) sense Target Labeling Assay is designed to generate amplified and biotynylated sense-strand DNA targets from the entire expressed genome. Protocol is optimized for the use with the GeneChip Sense Target (ST) Arrays, where the probes are distributed throughout the entire lenght of each transcript. At first $100 \mathrm{ng}$ of total
RNA was mixed with Poly-A RNA controls (GeneChip Eukaryotic Poly-A Control Kit, Affymetrix, Santa Clara, USA). Subsequently cDNA sysnthesis was performed with random hexamers tagged with a $\mathrm{T} 7$ promoter sequence (GeneChip WT cDNA Synthesis and Amplification Kit, Sub-kit 1: GeneChip WT cDNA Synthesis Kit; Affymetrix, Santa Clara, USA). The double-stranded cDNA was then used as a template to produce many copies of antisense cRNA (GeneChip WT cDNA Synthesis and Amplification Kit, Sub-kit 2: GeneChip WT cDNA Amplification Kit; Affymetrix, Santa Clara, USA). cRNA was treated with the cleanup procedure (GeneChip IVT cRNA, cDNA Cleanup Kit; Affymetrix, Santa Clara, USA). Subsequently cRNA yield was determined by spectrophotometric measurement (NanoDrop ND-1000; Thermoscientific). $10 \mu \mathrm{g}$ of cRNA was then used in the second cycle of cDNA synthesis. The random hexamers were used to prime riverse transcription of the cRNA to produce single-stranded DNA in the sense orientation (GeneChip WT cDNA Synthesis and Amplification Kit, Sub-kit 1: GeneChip WT cDNA Synthesis Kit; Affymetrix, Santa Clara, USA). During this step of the procedure, in order to reproducibly fragment ssDNA, dUTP was incorporated. Subsequently, ssDNA was then proceed with the cleanup procedure (GeneChip IVT cRNA, cDNA Cleanup Kit; Affymetrix, Santa Clara, USA). DNA yield was determined by spectrophotometric measurement (NanoDrop ND-1000; Thermoscientific).

$5 \mu \mathrm{g}$ of single-stranded DNA was treated with a combination of two enzymes: uracil DNA glycosidase (UDG) and apurinic/apyrimidinic endonuclease 1 (APE 1), specifically recognizing the dUTP nucleotides and breaking the DNA strand (GeneChip WT Terminal Labeling Kit; Affymetrix, Santa Clara, USA). Subsequently, fragmented DNA was labeled by terminal deoxynucleotidyl transferase (TdT) with the DNA Labeling Reagent, that was covalently linked to biotin (GeneChip WT Terminal Labeling Kit; Affymetrix, Santa Clara, USA). Then fragmented and labeled DNA was hybridized with the GeneChip Human Gene 1.0 ST Array (Affymetrix, Santa Clara, USA). Apart from DNA, hybridization cocktail included: Eukaryotic Hybridization Controls (bioB, bioC, bioD, cre), Control Oligonucleotide B2 (GeneChip Hybridization Control Kit; Affymetrix, Santa Clara, USA), 2x Hybridization Mix, DMSO, water (GeneChip Hybridization, Wash and Stain Kit- Hybridization Module; Affymetrix, Santa Clara, USA). Hybridization was performed in $450 \mathrm{C} / 60 \mathrm{rpm} / 17 \mathrm{~h} \pm 1 \mathrm{~h}$ at hybridization oven (GeneChip Hybridization Oven 640; Affymetrix, Santa Clara, USA).

After the hybridization, arrays were registered in GeneChip Operating Software (GCOS), and subsequently washed and stained with the use of GeneChip Fluidics Station 450 (Affymetrix, Santa Clara, USA) and FS450_0007 protocol (GeneChip Hybridization, Wash and Stain KitStain Module; Wash Buffer A; Wash Buffer B; Affymetrix, 
Santa Clara, USA). After the wash protocol was finished arrays were scanned with the GeneChip Scanner 3000 7G (Affymetrix, Santa Clara, USA) controlled by GeneChip Operating Software. The raw signal intensities were normalized (GeneChip Operating Software [GCOS] algorithm; Affymetrix); and the data were analyzed (Gene Chip DNA Analysis Software [GDAS], ver. 2.0) according to the Affymetrix GeneChip Expression Analysis Technical Manual (http://www.affymetrix.com). We detected a twofold change in differential gene expression between the normal and GCD2-homozygous (Arg124His mutation) samples. Expression of ECM-associated genes (TGFBIp, COL1A2, CTGF, and PAI-1) and H3K4 methyltransferase genes (MLL1, MLL2, ASH1L, SMYD3, SET7/9 and SETMAR) is presented as the $\log 2$ ratio. For statistical analysis, we used a two-tailed, unpaired Student's t-test (significance threshold $P<0.05$ ) to assess differences between the two cell types.

\section{ShRNA infection}

Lentiviruses containing human SET7/9, MLL1, and control shRNA, respectively, were purchased from Santa Cruz Biotechnology Inc. and used to infect corneal fibroblasts according to the manufacturer's instructions. Infected cells were selected using puromycin.

\section{Real-time quantitative reverse transcription PCR}

Total RNA was isolated from corneal fibroblasts by extraction in TRIZOL reagent (Invitrogen, Carlsbad, CA). Using the Power SYBR Green RNA-to-CT ${ }^{\text {mox }}$ 1-Step kit (Applied Biosystems, Foster City, CA, USA) and StepOnePlus $^{\text {Tw }}$ (Applied Biosystems, Foster City, CA, USA), mRNA expression of GAPDH, TGFBIp, collagen type I alpha 2 (COL1A2), plasminogen activator inhibitor-1 (PAI-1), and CTGF was measured according to the manufacturer's instructions. The PCR conditions for all the genes were as follows: $48{ }^{\circ} \mathrm{C}$ for $30 \mathrm{~min}, 95^{\circ} \mathrm{C}$ for $10 \mathrm{~min}$, then 40 cycles of $95{ }^{\circ} \mathrm{C}$ for $15 \mathrm{~s}$ and $60{ }^{\circ} \mathrm{C}$ for $1 \mathrm{~min}$. The results were based on cycle threshold $(\mathrm{Ct})$ values. We calculated the differences between the $\mathrm{Ct}$ values for the experimental genes and GAPDH (a reference gene) and graphed the results as the ratio of each RNA level to the calibrator sample level. The primers used for gene amplification were the following: TGFBI, 5'-CACAGTCTTTGCTCCCAC AA-3' (sense) and 5' -CTCCGCTAACCAGGATTTCA-3' (antisense); GAPDH, 5' -ATGGGGAAGGTGAAGGTCG3' (sense) and 5'-GGGGTCATTGATGGCAACAATA-3' (antisense); COL1A2, 5' - ATGGCTACCCAACTTGCCTT3'(sense) and 5' -ACAGCCTTTTTCAGGTTGCC-3' (antisense); PAI-1, 5' -CTGTCATAGTCTCAGCCCGC-3' (sense) and 5'-AAAGGACTGTTCCTGTGGGG-3' (antisense); and CTGF, 5'-GTGCATCCGTACTCCCAAAA-3' (sense) and $5^{\prime}$-ATCGGCCGTCGGTACATACT-3' (antisense). Three independent experiments were performed, and statistical analysis was carried out using Newman-Keuls multiple comparison tests.

\section{Chromatin immunoprecipitation for microarray analysis}

Chromatin immunoprecipitation (ChIP) assays were performed using the EpiQuik TM Chromatin Immunoprecipitation Kit (Epigentek) based on the protocol provided by the supplier (Epigentek Group Inc., Brooklyn, NY). Briefly, samples containing $4 \times 10^{6}$ human corneal fibroblasts were chemically cross-linked by the addition of fresh $11 \%$ formaldehyde solution (1:10 volumetric ratio of solution to sample) for $15 \mathrm{~min}$ at room temperature. The cells were then rinsed twice with $1 \times$ PBS, resuspended in CP3 lysis buffer containing a protease-inhibitor cocktail, and sonicated until the cross-linked chromatin was sheared to an average DNA fragment length of 200-1000 bp. One per cent of the sonicated lysate was used to quantify the total amount of DNA present in the different samples before immunoprecipitation (input). The sonicated samples were immunoprecipitated with H3K4me3 (Millipore, Millipore Corp., Bedford, MA) and H3K27me3 (Abcam, Cambridge, MA) antibodies using the EpiQuik TM Chromatin Immunoprecipitation Kit (Epigentek). Cross-linking between DNA and proteins was reversed by heating the samples at $65{ }^{\circ} \mathrm{C}$ for $15 \mathrm{~min}$ followed by Proteinase $\mathrm{K}$ digestion at $65^{\circ} \mathrm{C}$ for $1.5 \mathrm{~h}$. After cleaning on spin columns, the DNA was eluted in $10 \mathrm{mM}$ Tris-EDTA buffer.

\section{Chromatin immunoprecipitation microarray analysis}

The immunoprecipitated and input DNA was amplified using a whole-genome amplification kit (GenomePlex ${ }^{\circ}$ Complete Whole Genome Amplification Kit, Sigma, USA) according to the manufacturer's instructions. The amplified samples were purified using the QIAQuick PCR clean-up kit (Qiagen). Labeling reactions were performed with $4 \mu \mathrm{g}$ purified amplified DNA and a Bioprime labeling kit (Invitrogen) according to the manufacturer's instructions in a volume of $50 \mu \mathrm{l}$ with a modified dNTP pool containing $120 \mu \mathrm{M}$ each of dATP, dGTP, and dCTP; $60 \mu \mathrm{M}$ dTTP; and $60 \mu \mathrm{M}$ Cy5-dUTP (for the immunoprecipitated sample) or Cy3-dUTP (for the input sample). Labeled targets were subsequently purified using the QIAQuick PCR clean-up kit (Qiagen). Then, the dyelabeled DNA samples were purified and quantified using an ND-1000 spectrophotometer (NanoDrop Technologies, Inc., Wilmington, DE). After checking the labeling efficiency, each sample containing 2.5-5.0 $\mu \mathrm{g}$ cyanine 3labeled and cyanine 5-labeled DNA target was mixed and then resuspended with $2 \mathrm{X}$ hybridization buffer, Cot-1 DNA, Agilent 10X blocking agent, and de-ionized formamide. Before hybridization to the array, the $260-\mu \mathrm{l}$ hybridization mixtures were denatured at $95^{\circ} \mathrm{C}$ for $3 \mathrm{~min}$ and incubated at $37^{\circ} \mathrm{C}$ for $30 \mathrm{~min}$. The hybridization mixtures were centrifuged at 17,900 g for $1 \mathrm{~min}$ and directly 
pipetted onto the Human Promoter $1 \mathrm{M}$ microarray (Agilent Technology, USA). The arrays were hybridized at $65{ }^{\circ} \mathrm{C}$ for $40 \mathrm{~h}$ using an Agilent Hybridization oven (Agilent Technology, USA). The hybridized microarrays were washed according to the manufacturer's protocol (Agilent Technology, USA).

\section{Data acquisition and analysis}

The hybridization images were analyzed using an Agilent DNA Microarray Scanner (Agilent Technology, USA), and the data were quantified using the Agilent Feature Extraction software (Agilent Technology, USA). The preprocessing of the raw data and the normalization steps were performed using the Agilent Genomic Workbench software according to the manufacturer's instructions (Agilent, USA). The background-corrected intensity data were normalized by blank subtraction followed by intraarray LOWESS normalization. The peak detection was performed with Pre-defined Peak Shape detection v2.0 with $P$-value $<0.01$ as the threshold of significance for a non-parametric test and a peak-score $>5$ for an EVDbased score (based on T. Kaplan \& N. Friedman "Model-Based Analysis of High resolution Chromatin Immunoprecipitation" Technical Report 2006-11, School of Computer Science \& Engineering, Hebrew University 2006).

\section{Methylated DNA isolation assay-assisted CpG microarrays} Methylated DNA isolation assay (MeDIA)-assisted CpG microarray analysis was performed as described previously with slight modification [31]. Briefly, $1.0 \mu \mathrm{g}$ sonicfragmented genomic DNA from the test and control tissues, respectively, was incubated with $2 \mu \mathrm{g}$ recombinant MBD2bt protein for $4 \mathrm{~h}$ at $4{ }^{\circ} \mathrm{C}$ on a rocking platform. The enriched, methylated DNA was amplified using a whole-genome amplification kit (GenomePlex ${ }^{\circ}$, Sigma, USA) according to the manufacturer's protocol. The amplified DNA in the control and test samples was labeled with $\mathrm{Cy} 5$ and $\mathrm{Cy} 3$, respectively. The labeled samples were purified using a PCR purification kit (Qiagen, USA) and then co-hybridized to human promoter $1 \mathrm{M}$ microarrays (Agilent, Santa Clara, CA, USA) according to manufacturer's instructions.

\section{Microarray data analysis}

The hybridized images were analyzed using an Agilent DNA Microarray Scanner (Agilent Technology, USA), and the data were quantified using the Agilent Feature Extraction software (Agilent Technology, USA). The preprocessing of raw data and the normalization steps were performed using GeneSpring 7.3.1 (Agilent, USA). The background-corrected intensity data were normalized using the intensity-dependent LOWESS method to remove the dye bias within each array as recommended by the manufacturer (Agilent, USA). To select the multiple-probes-enriched target genes, the first level of data filtering involved the removal of all probes that showed only methylation ( $\geq$ twofold enrichment of the methylated fraction in test samples versus control samples) or nonmethylation ( $\leq$ twofold enrichment of the non-methylated fraction in test samples versus control samples). To increase the reliability of the results, we selected genes with probes in at least two adjacent probes.

\section{Chromatin immunoprecipitation assays}

ChIP assays were performed, and ChIP-enriched DNA was analyzed by qPCR as described previously [32]. Briefly, cells were fixed with $1 \%$ formaldehyde at $37{ }^{\circ} \mathrm{C}$ for $10 \mathrm{~min}$, washed with cold PBS containing protease inhibitors, and lysed in Tris ( $\mathrm{pH}$ 8.1) containing $1 \%$ SDS, $1 \mathrm{mM}$ PMSF, and complete protease-inhibitor cocktail. The cell lysates were sonicated into 500-bp chromatin fragments, diluted in ChIP dilution buffer, and immunoprecipitated overnight at $4{ }^{\circ} \mathrm{C}$ with the appropriate antibodies, with IgG control, or without antibody (no-antibody control). The next day, immune complexes were collected on ChIP-Grade Protein A/G Magnetic Beads (Thermo Fisher Scientific Inc., Rockford, IL USA), and the beads were washed to remove nonspecific binding. The DNA was eluted from the beads, the crosslinks were reversed, and the DNA was extracted. The ChIP-enriched DNA samples and the input DNA samples were analyzed by qPCR with SYBR reagent in a real-time PCR machine (ABI 7300; Applied Biosystems) using primers specific for the TGFBI, Col1a1, CTGF, or PAI-1 promoters spanning Smad binding elements (SBEs). All reactions were performed in triplicate in a final volume of $20 \mu \mathrm{l}$. Dissociation curves were run to detect nonspecific amplification, and we confirmed that single products were amplified in each reaction. The qPCR data were analyzed using the $2^{-\Delta \Delta C t}$ method, as described previously [32], and normalized with the input samples. The results were expressed as the fold change relative to the control. In all of the experiments, we verified that the ChIP samples obtained with specific antibodies exhibited significant enrichment relative to the IgG or no-antibody controls. The primers used for the ChIP-enriched DNA were: TGFBIp, 5' -TACCTGCCTTGAGCTCCTCC-3' (sense) and 5' -GT GGGGTCCTCACCTTGGTA-3' (antisense); COL1A2, 5' CTCCGACGTGTCCCATAGTG-3' (sense) and 5' -CTTT TGAGGCTTTCAAGGGG-3' (antisense); PAI-1, 5' ${ }^{\prime}$-AGT CTGGACACGTGGGGAGT-3' (sense) and 5'-GCCAGC CACGTGATTGTCTA-3' (antisense); and CTGF, 5'-TC TGTGAGCTGGAGTGTGCC-3' (sense) and 5' -ACAGG GACATTCCTCGCATT-3' (antisense).

\section{Western blotting}

Cell lysates were fractionated by SDS-PAGE and transferred to PVDF membranes. The blocked membranes were 
incubated with the appropriate antibody, and the immunoreactive bands were visualized with a chemiluminescent reagent as recommended by Amersham Biosciences, Inc.

\section{Statistical analysis}

All experiments were repeated at least three times. Data are presented as the mean \pm standard error (SE), and statistical comparisons between groups were performed by one-way ANOVA followed by Tukey's test.

\section{Results}

Transcription and extracellular-secretion levels of TGFBIp were high in wild-type cells compared with those in GCD2 homozygous cells and were related to H3K4me3 levels but not to DNA methylation

Not only mutation of TGFBIp gene, but also regulation of TGFBIp expression is very important cause of in GCD2.

To investigate the mechanisms of TGFBIp gene expression, we first analyzed TGFBIp mRNA transcription levels in wild-type and GCD2-homozygous corneal fibroblasts (Fig. 1a, b). The TGFBIp mRNA transcription levels were much higher in the wild-type cells compared with those in the GCD2-homozygous cells (Fig. 1c). Moreover, the TGFBIp protein levels secreted into the culture supernatant were much higher for the wild-type cells compared with those for the GCD2-homozygous cells. The respective cell lysates from the wild-type and GCD2-homozygous cells showed similar levels of TGFBIp (Fig. 1d), however, suggesting that mutant TGFBIp accumulates in GCD2 corneal fibroblasts as a result of either impaired degradation or delayed extracellular secretion [33], causing similar intracellular TGFBIp levels in the wild-type and GCD2 homozygote cells despite the differences in TGFBIp mRNA transcription and extracellular-secretion levels.

We next examined whether the difference in TGFBIp gene expression was associated with changes in either the active epigenetic marker $\mathrm{H} 3 \mathrm{~K} 4 \mathrm{me} 3$ or the repressive epigenetic marker $\mathrm{H} 3 \mathrm{~K} 27 \mathrm{me} 3$ in the $\mathrm{H} 3$ promoter. As

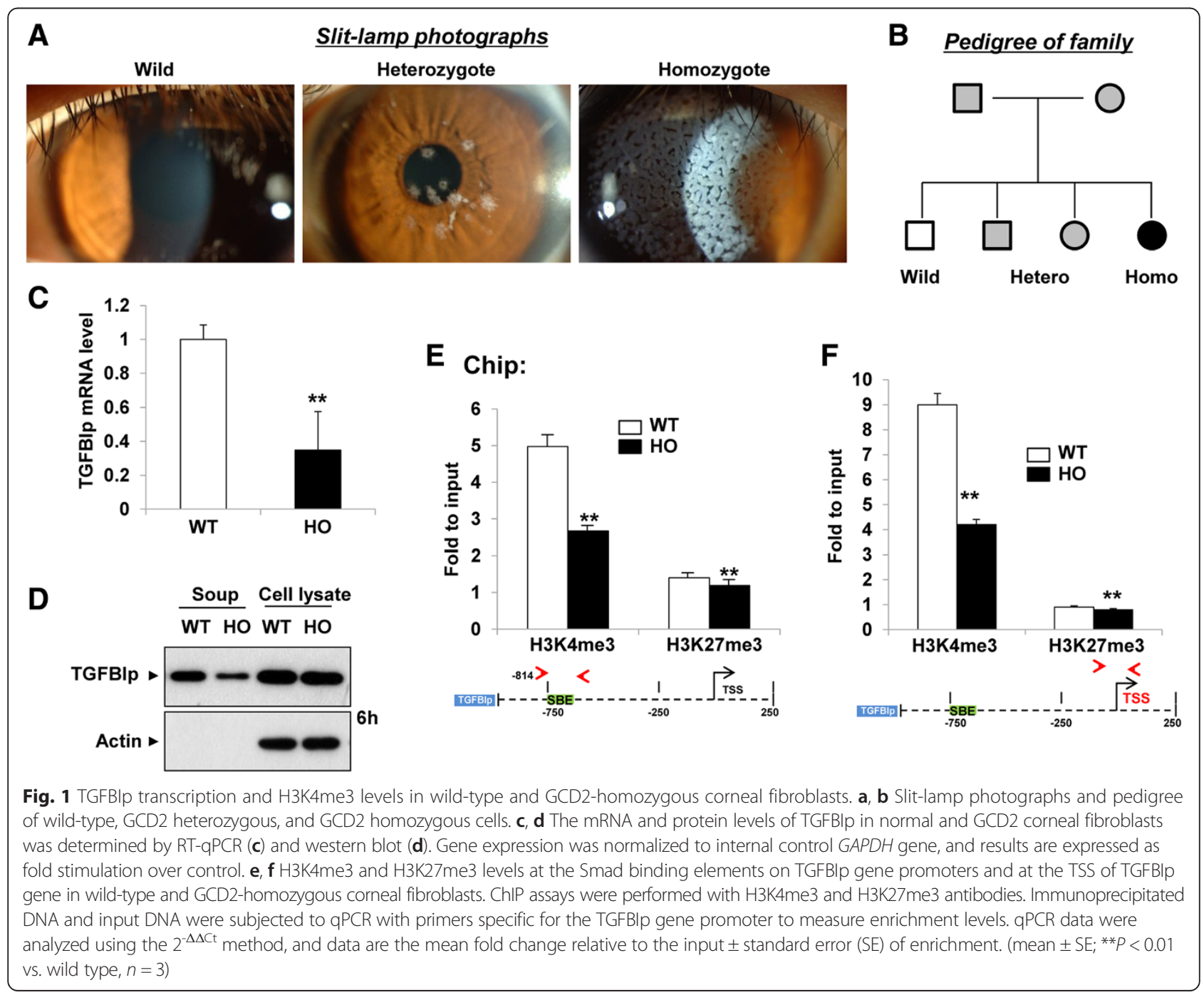


shown in Fig. 1e, f, the H3K4me3 levels in the Smad Binding Element (SBE) of the TGFBIp promoter and in the TSS of the TGFBIp gene were much higher in the wildtype cells compared with those in the GCD2-homozygous cells, similar to the TGFBIp mRNA transcription levels. The H3K27me3 levels were very low in both the wild-type and the GCD2-homozygous cells, however, and were not different between the two cell types. To further confirm the association between H3K4me3 levels and TGFBIp mRNA transcription levels, we analyzed wild-type $(n=3)$, GCD2-heterozygous $(n=1)$, and GCD2-homozygous $(n=3)$ corneal fibroblasts. As shown in Additional file 1: Figure S1A-C, the TGFBIp mRNA transcription levels and extracellular secreted-protein levels were much higher in the wild-type cells compared with those in the GCD2 cells and were strongly related to the H3K4me3 levels. The intracellular TGFBIp protein levels were not related to the mRNA or H3K4me3 levels in the GCD2 cells, however (Additional file 1: Figure S1D).

We next performed a ChIP on chip assay with H3K4me3-specific and H3K27me3-specific antibodies in wild-type $(n=2)$ and GCD2-homozygous $(n=2)$ primary corneal fibroblasts. As shown in Fig. 2a and b, the H3K4me3 levels in the TGFBIp promoter region and the downstream of transcription start site were high in the wild-type cells compared with those in the GCD2 cells, but the H3K27me3 levels were very low in both the wild-type and the GCD2 cells and were not different between the two cell types. In contrast, DNA methylation levels in the TGFBIp promoter region were very low and were not different between the two cell types (Fig. 2c). Collectively, these results suggest that the mRNA transcription and extracellular-secretion levels of TGFBIp were high in the wild-type cells compared with those in the GCD2-homozygous cells and were related to the H3K4me3 levels but not to DNA methylation over the TGFBI locus.

\section{TGF $\beta 1$ increased the expression of TGFBIp and} ECM-associated genes and Smad3 recruitment to the TGFBIp and ECM-associated gene promoters

We examined whether TGFBIp expression could be induced by TGF $\beta 1$ treatment in corneal fibroblasts. As shown in Fig. 3a and b, TGFBIp protein and mRNA

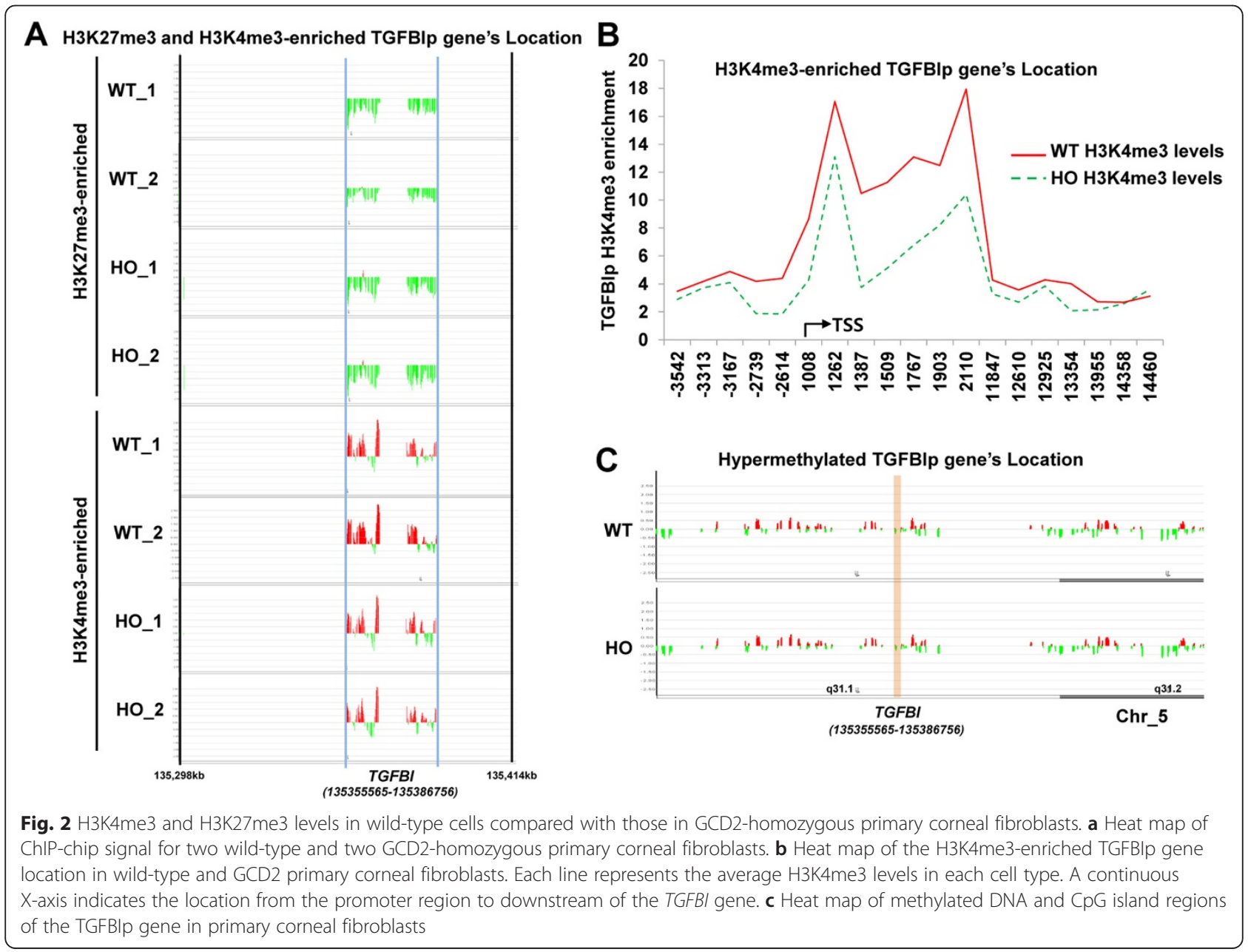




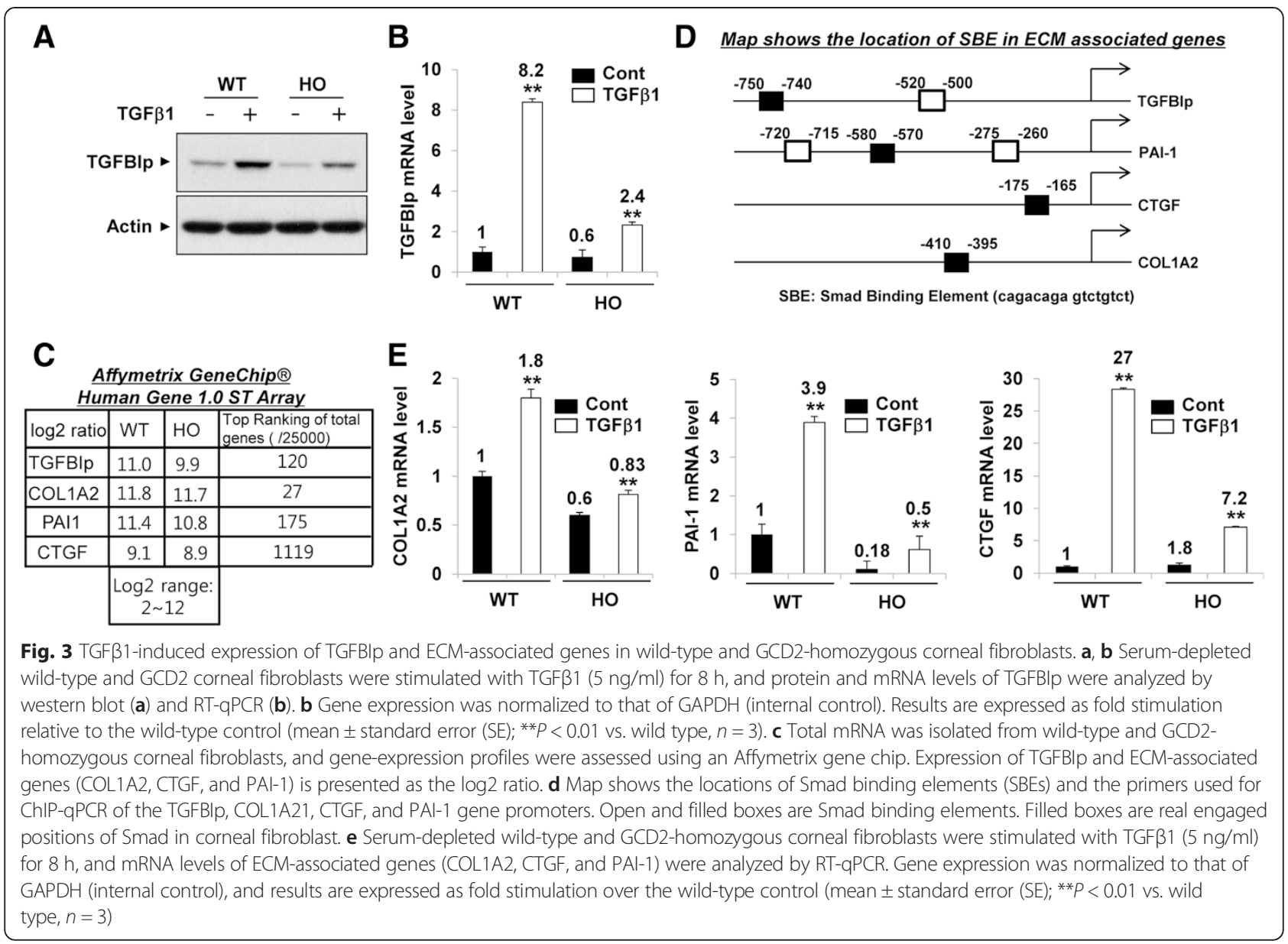

levels were significantly increased by TGF $\beta 1$ in the wildtype cells, and the GCD2-homozygous cells showed much lower TGFBIp levels compared with the wild-type cells. Furthermore, As shown in Fig. 3c, the ECMassociated genes for COL1A2, CTGF, and PAI-1 were highly expressed together with TGFBIp in both the wildtype and the GCD2 corneal fibroblasts. The promoters of those ECM-associated genes contain the SBE, which is a TGF $\beta 1$ downstream effector-signaling molecule (Fig. 3d). Therefore, we examined whether the ECMassociated gene expression could be induced by TGF $\beta 1$ treatment in corneal fibroblasts. As shown in Fig. 3e, the COL1A2, PAI-1, and CTGF mRNA levels were significantly increased by TGF $\beta 1$ in wild-type corneal fibroblasts, whereas the GCD2 homozygous cells showed much smaller increases in ECM-associated gene expression levels under same conditions (Fig. 3e).

Next, we examined whether TGF $\beta 1$ altered the Smad3 occupancy on the promoters of the TGFBIp, COL1A2, PAI-1, and CTGF genes using ChIP assays with Smad3 antibodies. As shown in Fig. 4, Smad3 recruitment was significantly increased on the TGFBIp, COL1A2, PAI-1, and CTGF promoters after TGF $\beta 1$ stimulation in the
GCD2-homozygous cells relative to that in the wild-type cells (Fig. 4). The GCD2-homozygous cells showed very small increases in Smad3 recruitment to the TGFBIp and ECM-associated gene promoters, however (Fig. 4), suggesting that TGF $\beta 1$ increases the expression of TGFBIp and ECM-associated genes by increasing Smad3 recruitment to the TGFBIp and ECM-associated gene promoters in corneal fibroblasts.

\section{TGF $\beta 1$ enhanced $H 3 K 4 m e 1 / 3$ levels on the promoters of TGFBIp and ECM-associated genes}

To investigate whether TGF $\beta 1$ could alter promoter levels of H3K4me, we performed ChIP assays with H3K4me1 or H3K4me3 antibodies. As shown in Fig. 5a and b, TGF $\beta 1$ strongly increased H3K4me1 and H3K4me3 levels on the TGFBIp and ECM-associated gene promoters in the wildtype corneal fibroblasts. But only weakly increased those levels in the GCD2-homozygous cells under the same conditions (Fig. 5a, b). The increases in promoter H3K4me1/3 levels were correlated with the increased expression of the associated genes induced by TGF $\beta 1$. In contrast, levels of the repressive epigenetic marker H3K27me3 decreased in TGF 31 -stimulated wild- 


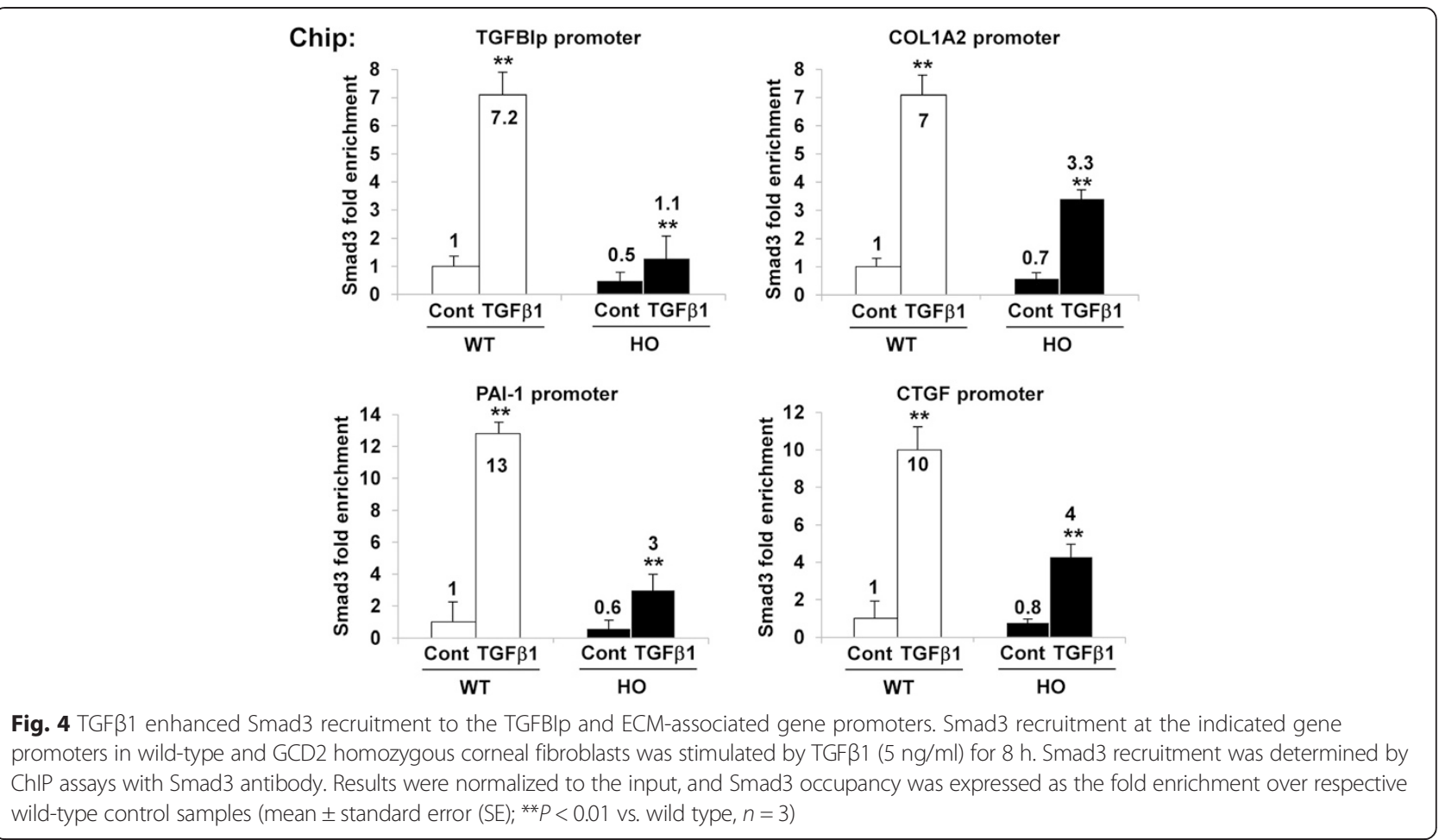

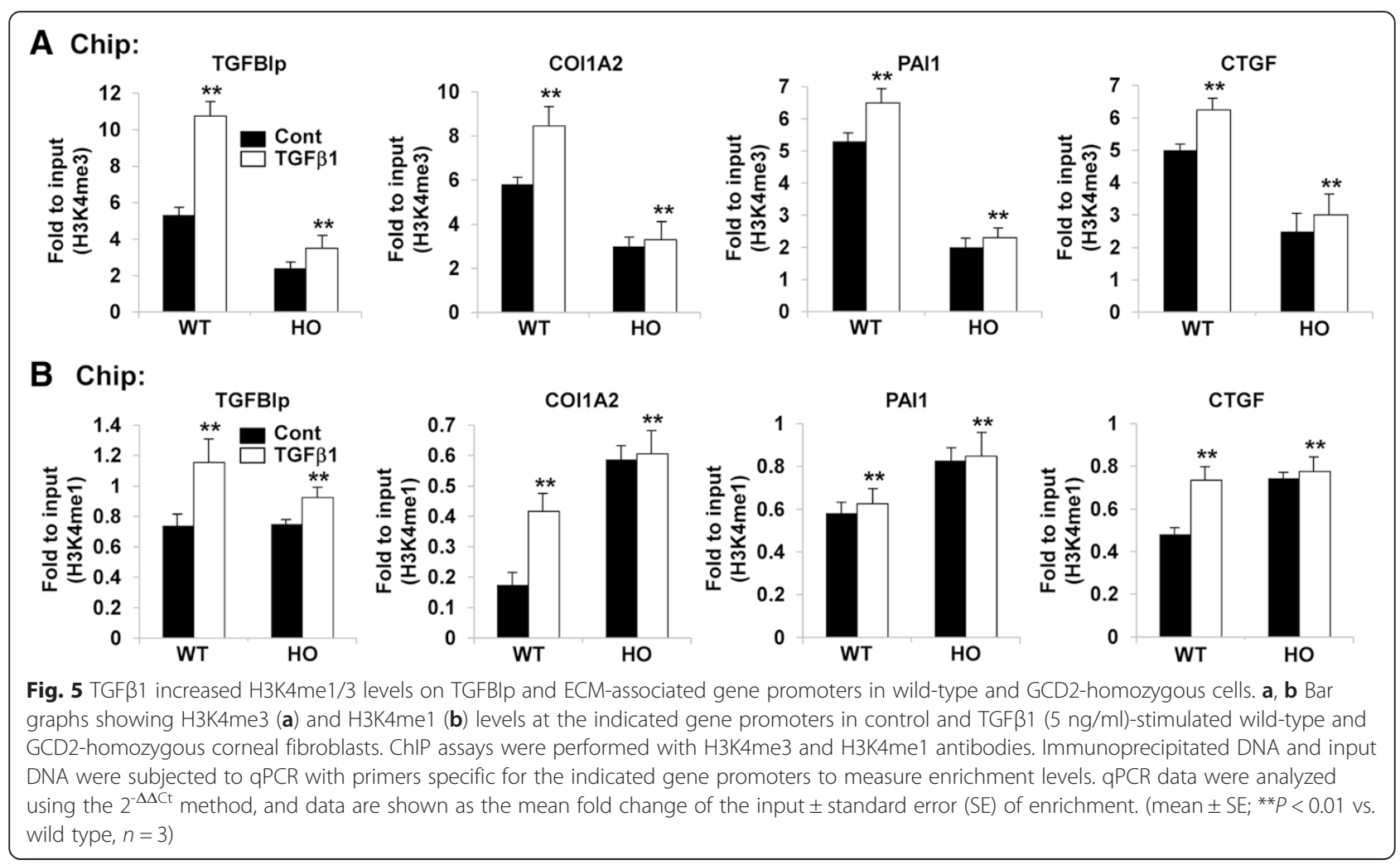


type corneal fibroblasts (Additional file 1: Figure S2A), but there was no significant effect of TGF 31 on H3K27me3 levels in the GCD2-homozygous cells (Additional file 1: Figure S2A). Furthermore, levels of H3K9me3, another repressive epigenetic marker, were not significantly changed in either the wild-type or the GCD2-homozygous cells under same conditions (Additional file 1: Figure S2B), suggesting that increases in $\mathrm{H} 3 \mathrm{~K} 4 \mathrm{me} 1 / 3$ and decreases in H3K27me3 on the target-gene promoters may be involved in the TGF 1 -induced up-regulation of TGFBIp and ECM-associated genes in corneal fibroblasts.

TGF $\beta 1$ increased MLL1 recruitment to the TGFBIp and ECM-associated gene promoters

To identify genes that regulate the $\mathrm{H} 3 \mathrm{~K} 4 \mathrm{me} 1 / 3$ induced by TGF $\beta 1$, we analyzed the expression of H3K4methyltransferase genes in wild-type and and GCD2 corneal fibroblasts by microarray analysis. As shown in Fig. 6a, MLL1, an H3K4 tri-methyltransferase, and SET7/ 9, an H3K4 mono- methyltransferase, were expressed in wild-type and GCD2 cells. Next, we examined whether TGF $\beta 1$ alters the MLL1 occupancy on the promoters of the TGFBIp, COL1A2, PAI-1, and CTGF genes using ChIP assays with MLL1 antibodies. As shown in Fig. 6b and c, MLL1 recruitment was significantly increased in the TGFBIp, COL1A2, PAI-1, and CTGF promoters after TGF $\beta 1$ stimulation in the wild-type corneal fibroblasts (Fig. 6b, c). The GCD2 homozygous cells showed very weak increased recruitment of MLL1 to the TGFBIp and ECM-associated gene promoters (Fig. 6b, c). The MLL1 recruitment pattern was quite similar to the pattern of increased H3K4me3 (Fig. 5a), suggesting a key role for MLL1 in the TGF 31 -mediated increase in H3K4me3 during the induction of the TGFBIp and ECM genes.

\section{MLL1 and SET7/9 knockdown attenuated the TGF $\beta 1$-induced expression of the TGFBIp and ECM-associated genes}

Corneal fibroblasts were first infected with lentivirus containing shRNA targeting MLL1 (shMLL1) or control shRNA (shCont), and protein levels were analyzed by western blot. As shown in Fig. 7a, MLL1 protein levels were significantly reduced in the wild-type and GCD2 cells infected with shMLL1 lentivirus compared with those in wild-type and GCD2 cells infected with shCont lentivirus. We next examined whether MLL1 shRNA can affect H3K4me3 in corneal fibroblasts, because previous studies showed that MLL1 regulates H3K4me3 in embryonic fibroblasts and immune cells [34-36]. Global H3K4me3 levels were significantly reduced in both the

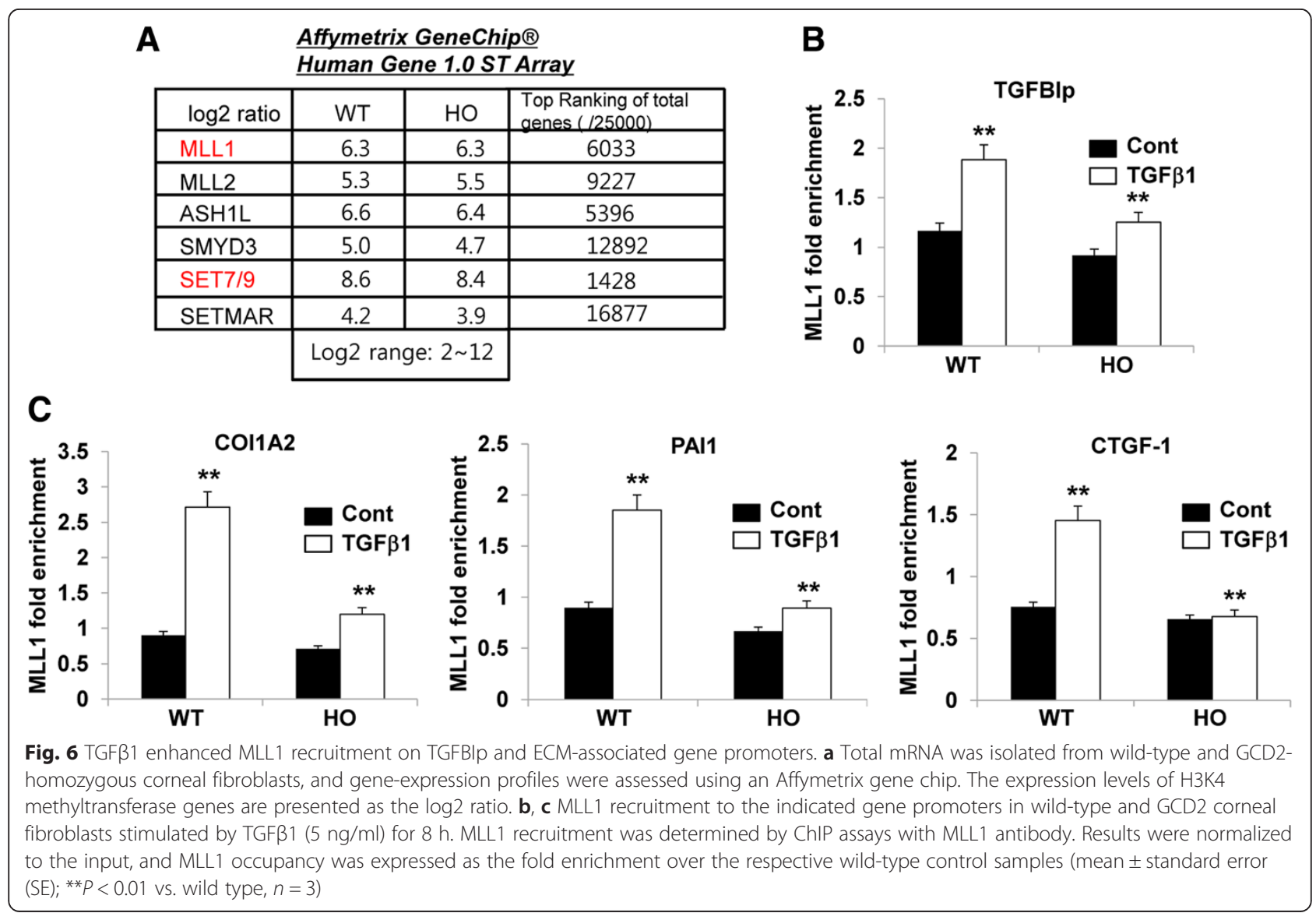




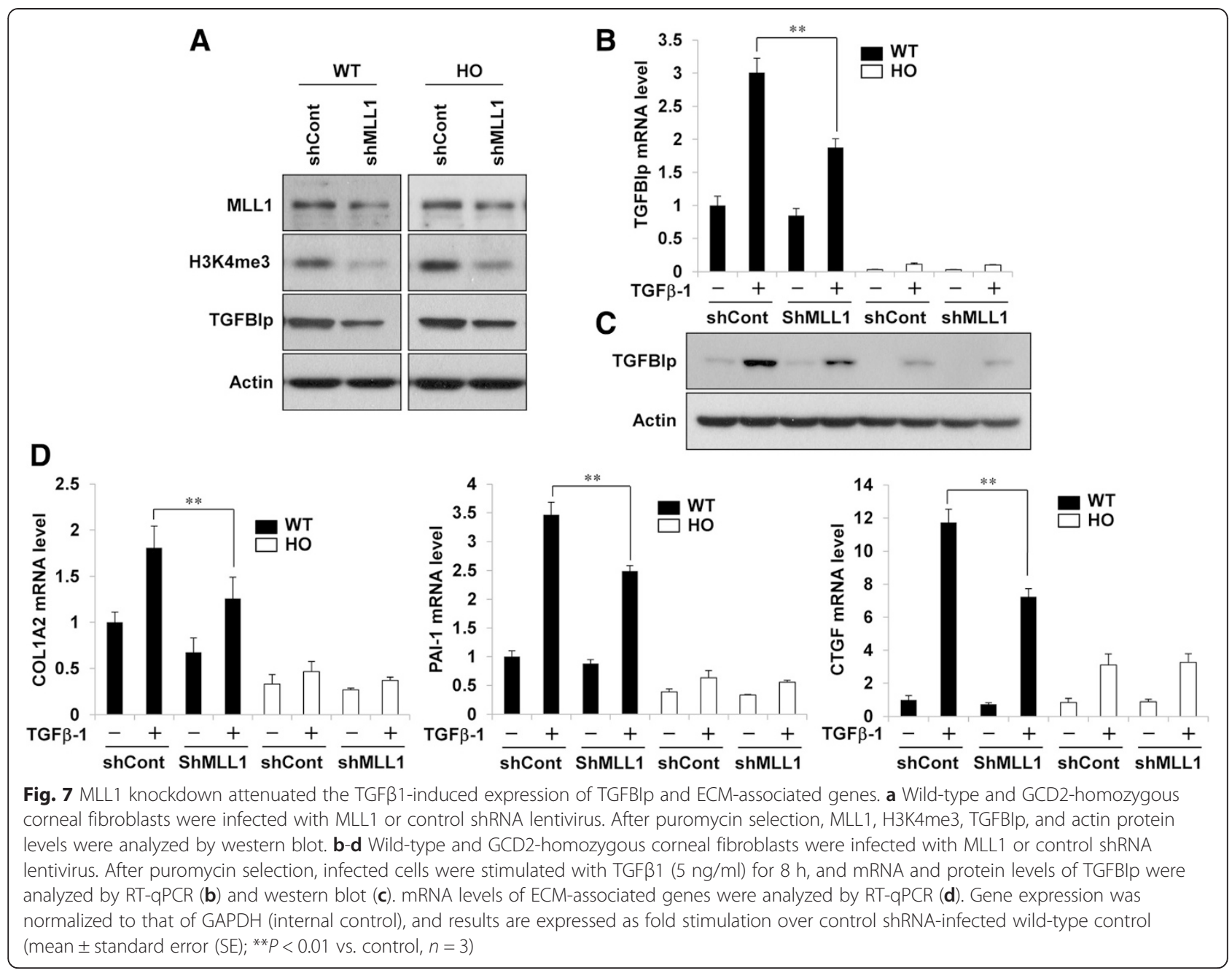

wild-type cells and the GCD2 cells infected with shMLL1 lentivirus (Fig. 7a). Also, TGFBIp protein levels were reduced in cells infected with shMLL1 lentivirus. Furthermore, shMLL1 significantly attenuated TGF $\beta 1$ induced TGFBIp protein and mRNA levels in wild-type corneal fibroblasts compared with shCont (Fig. 7b, c). The GCD2-homozygous cells showed very weak TGFBIp expression and inhibition induced by TGF $\beta 1$ and shMLL1, respectively (Fig. 7b, c). TGF $\beta 1$-induced COL1A2, PAI-1, and CTGF mRNA levels were inhibited by shMLL1 (Fig. 7d), whereas no significant increase and decrease in response to TGF $\beta 1$ and shMLL1 were observed in the GCD2-homozygous cells (Fig. 7d), suggesting a key role for MLL1 in modulating TGF $\beta 1$ responses in corneal fibroblasts.

To investigate the role of SET7/9 in the expression of the TGFBIp and ECM genes, cells were infected with shSET7/9 or shCont lentivirus, and protein levels were analyzed by western blot. As shown in Additional file 1: Figure S3A, total H3K4me1 levels were clearly decreased in the SET7/9 knockdown cells compared with the shCont cells. In addition, the TGFBIp protein levels were inhibited in the cells infected with shSET7/9 (Additional file 1: Figure S3A). Furthermore, TGF $\beta 1$-induced TGFBIp protein and mRNA levels were inhibited by shSET7/9 (Additional file 1: Figure S3B, C) in wild-type corneal fibroblasts. The GCD2-homozygote cells showed very weak TGFBIp expression and inhibition induced by TGF $\beta 1$ and shSET7/9, respectively (Additional file 1: Figure S3B, C). TGF 31 -induced COL1A2, PAI-1, and CTGF mRNA levels were also partially inhibited by shSET7/9 lentivirus infection (Additional file 1: Figure S3D), whereas no significant increase and decrease induced by TGF $\beta 1$ and shSET7/9, respectively, was observed in the GCD2-homozygous cells (Additional file 1: Figure S3D). Thus, SET7/9 likely partially mediates the induction of H3K4me1 by TGF $\beta 1$ in corneal fibroblasts. Collectively, these results suggest that MLL1 and SET7/9 are involved in basal TGFBIp expression and TGF $\beta 1$-induced TGFBIp and ECM-associated gene expression. 
MLL1 and SET7/9 knockdown attenuated the TGF $\beta 1$-induced increase in H3K4me1/3 levels on the promoters of the TGFBIp and ECM-associated genes We tested whether the knockdown of MLL1 and SET7/9 can decrease active H3K4me1/3 markers on the promoters of the TGFBIp and ECM-associated genes. The knockdown of MLL1 and SET7/9 significantly decreased TGFß1-mediated H3K4me3 (Fig. 8a) and H3K4me1 (Additional file 1: Figure S4) levels on the TGFBIp, COL1A2, PAI-1, and CTGF promoters. These findings show the mediatory roles of MLL1 and SET7/9 in TGF 31 induced epigenetic events on the promoters of the TGFBIp and ECM genes and in the subsequent expression of those genes.

To better understand the roles of MLL1 and SET7/9 in TGFBIp expression, cells were co-infected with shMLL1 and shSET7/9, and TGFBIp mRNA levels were analyzed by RT-qPCR. As shown in Fig. 8b, shMLL1 and shSET7/9 co-infection completely inhibited TGF 31 -induced TGFBIp mRNA levels compared with shMLL1 or shSET7/9 single infection, providing evidence that both MLL1 and SET7/9 mediate TGF $\beta 1$-induced TGFBIp expression by regulating H3K4me1/3.

\section{Discussion}

In GCD2 pathogenesis, mutations in the TGFBI gene important, but the regulation of TGFBIp expression is also very important. We confirmed that mRNA transcription and extracellular-secretion levels of TGFBIp were high in wild-type corneal fibroblasts compared with those in GCD2-homozygous cells. The expression pattern was strongly related to H3K4me3 levels but not

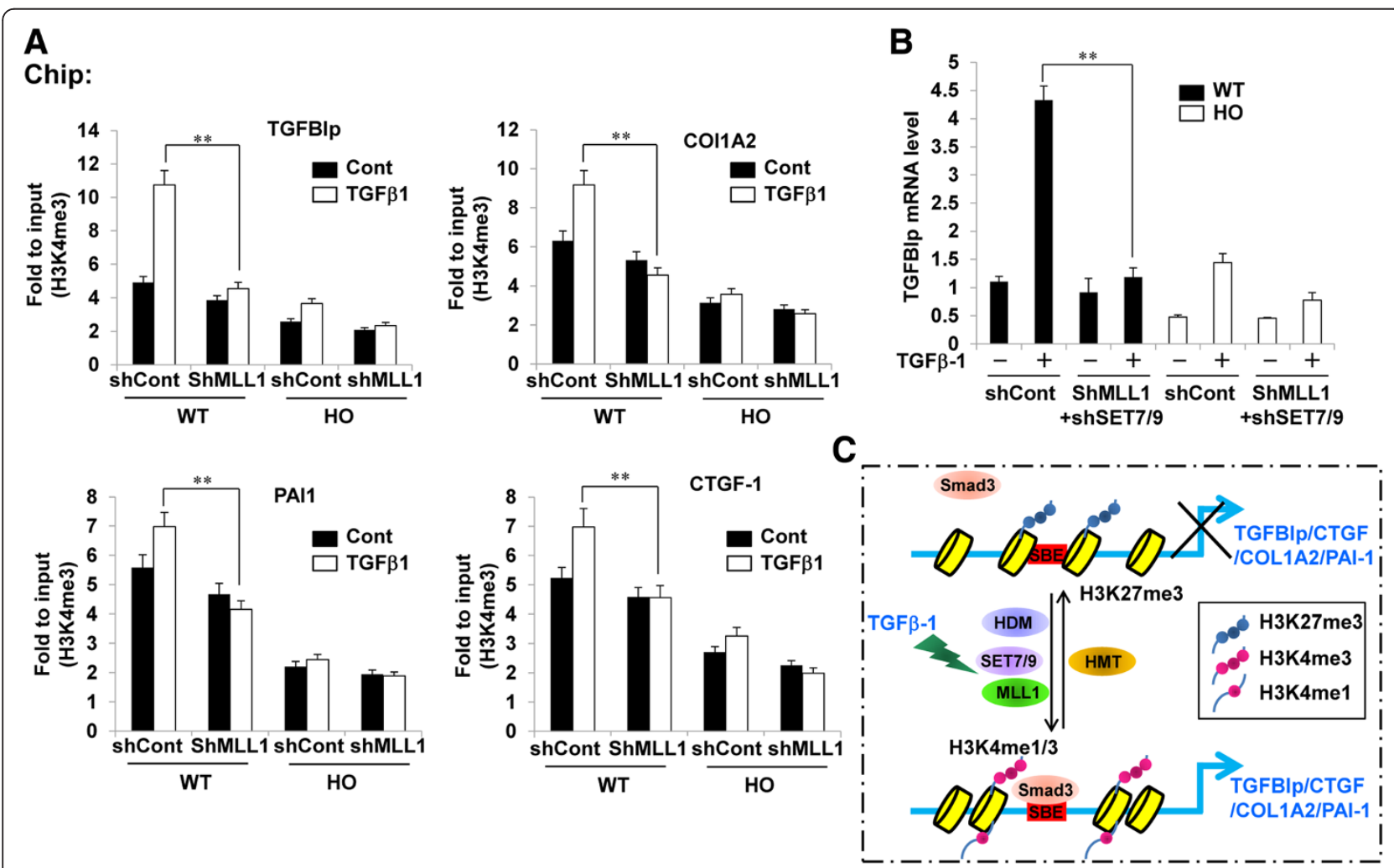

Fig. 8 MLL1 knockdown attenuated TGFß1-induced increases in H3K4me3 on the promoters of TGFBlp and ECM-associated genes. a Wild-type and GCD2-homozygous corneal fibroblasts were infected with MLL1 or control shRNA lentivirus. After puromycin selection, infected cells were stimulated with TGF- $\beta 1$ ( $5 \mathrm{ng} / \mathrm{ml})$ for $8 \mathrm{~h}$, and H3K4me3 levels at the indicated gene promoters were analyzed. ChIP assays were performed with H3K4me3 antibody. Immunoprecipitated DNA and input DNA were subjected to qPCR with primers specific for the indicated gene promoters to measure enrichment levels. qPCR data were analyzed using the $2^{-\Delta \Delta C t}$ method, and data are presented as mean fold change of the input \pm standard error (SE) of enrichment. (mean $\pm S E$; ${ }^{*} P<0.01$ vs. control, $n=3$ ). b Wild-type and GCD2-homozygous corneal fibroblasts were double infected with MLL1 and SET7/9 or control shRNA lentivirus. After puromycin selection, infected cells were stimulated with TGF $\beta 1$ ( $5 \mathrm{ng} / \mathrm{ml})$ for $8 \mathrm{~h}$, and mRNA levels of TGFBlp were analyzed by RT-qPCR. Gene expression was normalized to that of GAPDH (internal control), and results are expressed as fold stimulation over control shRNA-infected wild-type control (mean \pm standard error (SE); ${ }^{*} P<0.01$ vs. control, $n=3$ ) (c) A model showing that in corneal fibroblasts reaching the quiescent state, transcriptional repression of TGFBIp and ECM-associated genes occurs through heterochromatin formation under no stimulation. Lysine 27 from histone H3 (H3K27) near Smad binding elements (SBEs) within promoters was tri-methylated. During TGF 31 stimulation, MLL1 and SET7/9 were activated, and their recruitment to SBEs was increased and recruitment of Smad to SBEs was increased. H3K4me1/3 became more methylated, and H3K27me3 was de-methylated on the gene promoters, creating a favorable environment for Smad3 binding 
to DNA methylation over the TGFBI locus. Intracellular TGFBIp levels were similar in wild-type and GCD2homozygous cells, although TGFBIp mRNA transcription and extracellular secreted-protein levels were much lower in GCD2-homozygous cells compared with wildtype cells, because mutant TGFBIp can accumulate in GCD2 corneal fibroblasts as a result of either impaired degradation or delayed extracellular secretion [33]. The abnormal conditions within GCD2 cells caused by mutant-TGFBIp accumulation may weaken responses to exogenous stimuli such as TGF $\beta 1$ compared with those in wild-type cells.

Alternately, the observed differences in histone modification in GCD2 fibroblasts compared to normal cells may be due to the reduced expression of TGFBI in the mutant cells. Much less TGFBIp is secreted from mutant than from wild type cells. However, intracellular TGFBIp is at similar levels in both cell types. Given the reduced secretion of the protein in mutant cells, less transcription is required to maintain intracellular protein levels and negative feedback would suppress transcription in mutant relative to wild type cells. Thus, it is expected that levels of H3K4me3, a well-known marker of active transcription, would be reduced at the promoter of the TGFBI gene. Our data show that the transcription of TGFBI and associated ECM genes is increased upon treatment with TGF $\beta 1$ and that H3K4me3 levels again correlate with expression. These effects are reduced, but not eliminated, in mutant cells. The association of active histone modifications with levels of transcription may not represent proof of a causative link. Rather, it is more likely that the accumulation of TGFBIp is driving the altered transcription pattern in mutant cells.

In our study, we found that TGF $\beta 1$ can up-regulate TGFBIp and ECM-associated genes such as COL1A2, PAI-1, and CTGF in corneal fibroblasts. TGFBIp expression induced by TGF $\beta 1$ was seen previously in corneal fibroblasts, but our study is the first to demonstrate increases in the expression of other ECM genes that may coagulate with TGFBIp in the corneas of patients with GCD2. We observed that TGF $\beta 1$ increased Smad3 recruitment to the TGFBIp and ECM-associated gene promoters in corneal fibroblasts. TGFBIp and ECMassociated genes have conserved SBEs in their promoters, suggesting that gene expression could be similarly regulated under conditions such as TGF $\beta 1$ stimulation.

We examined changes in key epigenetic chromatin markers, including H3K27me3 and H3K4me1/3 levels, on the TGFBIp and ECM-associated gene promoters. Our results showed that those markers, along with H3K27 histone de-methylases (HDMs) and the MLL1 and SET7/9 HMTs, are involved in the TGF $\beta 1$-induced up-regulation of TGFBIp and ECM-associated genes in corneal fibroblasts. We showed that the up-regulation of H3K4me1/3 markers usually associated with active chromatin occurs in parallel with the down-regulation of H3K27me3 by TGF $\beta 1$, suggesting that those regulatory mechanisms can further contribute to the increased gene expression. Recent studies showed that methylated H3K4 correlates with transcriptionally competent chromatin and is associated with active genes [25-27], supporting our observation that TGF $\beta 1$ increased the expression of the TGFBIp and ECM genes in corneal fibroblasts and that such expression was positively correlated with increased H3K4meland H3K4me3 levels on the associated promoters.

Increasing evidence shows that H3K27me3 markers are recognized by polycomb protein and generally correlate with gene silencing and transcriptional repression $[26,37,38]$. Our results showed for the first time that TGF $\beta 1$ decreased H3K27me3 levels on the TGFBIp and ECM gene promoters and that those levels were inversely correlated with the increased expression of the genes, further supporting the notion that a relief of transcriptional repression caused by decreases in repressive chromatin histone modifications may contribute to the increased expression of TGFBIp and ECM genes induced by TGF $\beta 1$. Collectively, our observations show that increases in H3K4me1/3 and decreases in H3K27me3 at the TGFBIp and ECM gene promoters in the presence of TGF $\beta 1$ in corneal fibroblasts are correlated with the TGF $\beta 1$-induced up-regulation of those genes.

Furthermore, we showed not only an increase in the recruitment of the H4K4 HMTs MLL1 and SET7/9 at the TGFBIp and ECM gene promoters but also a decrease in the recruitment of H4K27 HMT in corneal fibroblasts stimulated by TGF $\beta 1$, suggesting that the H4K4 HMT MLL1 is involved in the TGF $\beta 1$-induced up-regulation of the TGFBIp and ECM genes in corneal fibroblasts. This was supported by our observations that MLL1 and SET7/9 gene silencing partially, but significantly, blocked the expression of the TGF $\beta 1$-induced TGFBIp and ECM genes. The knockdown of MLL1 and SET7/9 with shRNAs could decrease global H3K4me1 and $\mathrm{H} 3 \mathrm{~K} 4 \mathrm{me} 3$ levels, suggesting that $\mathrm{H} 3 \mathrm{~K} 4 \mathrm{me} 1 / 3$ mediated by MLL1 and SET7/9 plays a key role in TGFBIp and ECM-associated gene expression and that MLL1 and SET7/9 are potential therapeutic targets for the treatment of ECM-accumulation disorders such as GCD2. The observed increases in H3K4me3 might synergize with other complementary events occurring on the TGFBIp and ECM-associated gene promoters, such as increases in H3K4me1 and decreases in H3K27me3, to enhance TGFBIp and ECM gene expression in response to TGF $\beta 1$. In addition, the HMTs and HDMs, as well as the HATs and histone deacetylases, regulating the complementary chromatin markers may also play cooperative roles. Additional studies are needed to assess those factors, including the role of the HMTs that mediate H3K4me1/3 and 
H3K27me3. It was suggested that the H3K4me1/3 mediated by MLL1 and SET7/9 functions in transcriptional activation by competing with histone deacetylases to enhance H3-K9 acetylation and prevent H3-K9 methylation [39, 40]. MLL1 and SET7/9 can methylate nonhistone proteins including p53 [41, 42], DNMT1 [43], TAF10 [44], and p65 [45, 46]. Furthermore, MLL1 and SET7/9 could regulate a subset of TNF $\alpha$-induced, NF-kBdependent inflammatory genes in monocytes [47] and the high glucose-induced expression of NF- $\mathrm{B}$ B p65 and inflammatory genes in endothelial cells [48], indicating the diverse physiologic roles of MLL1 and SET7/9 in gene transactivation. Similarly, MLL1 and SET7/9 may also methylate other non-histone proteins and possibly even Smads in corneal fibroblasts. Further studies are needed to evaluate those possibilities. In the future, analogous approaches should be explored to examine epigenetic mechanisms of regulating the expression of other matrix genes in corneal epithelial cells exposed to TGF $\beta 1$. Epigenetic mechanisms may affect ECM expression by regulating the expression of metalloproteases and other proteases, because histone modification has been shown to regulate metalloproteases in some cells [49]. Taken together, our results show that TGF $\beta 1$ can promote significant changes in $\mathrm{H} 3 \mathrm{~K} 4$ and $\mathrm{H} 3 \mathrm{~K} 27$ methylation on gene promoters in corneal fibroblasts that correlate with parallel increases in the expression of genes related to ECM accumulation and GCD2 pathogenesis.

\section{Conclusions}

In summary, our findings clearly show that epigenetic histone methylation modulates the expression of TGFBIp and ECM genes in corneal fibroblasts. In corneal fibroblasts reaching the quiescent state, the transcriptional repression of TGFBIp and ECM-associated genes occurs through heterochromatin formation with no external stimulation. H3K27 residues near SBEs within promoters are tri-methylated. During TGF $\beta 1$ stimulation, MLL1 and SET7/9 are activated, increasing their recruitment to SBEs. H3K4me1/3 becomes more methylated, and H3K27me3 becomes de-methylated on gene promoters, creating a favorable environment for Smad3 binding, resulting in the increased expression of TGFBIp and ECM-associated genes (Fig. 8c). The effective epigenetic regulation of histone $\mathrm{H} 3 \mathrm{~K} 4 \mathrm{me}$ during the TGF 31 mediated expression of TGFBIp and ECM genes in corneal fibroblasts could be used to create cornea-protective therapies for granular corneal dystrophy.

\section{Additional file}

Additional file 1: Figure S1. TGFBIp transcription was high in wild-type cells compared with that in GCD2 corneal fibroblasts and was related to H3K4me3 levels. (A) H3K4me3 levels at the Smad binding elements of
TGFBlp gene promoters in wild-type, GCD2-heterozygous, and GCD2homozygous corneal fibroblasts. ChIP assays were performed with H3K4me3 antibody. (mean \pm SE). (B) mRNA levels of TGFBIp in corneal fibroblasts were determined by RT-qPCR. (C, D) Protein levels of TGFBIp in cell lysates or culture supernatants were determined by western blot. Figure S2. TGFb1 had a minor effect on H3K27me3 and H3K9me3 modification on the TGFBIp and ECM-associated gene promoters. Bar graphs showing H3K27me3 (A) and H3K9me3 (B) levels on the indicated gene promoters in control and TGFb1 $(5 \mathrm{ng} / \mathrm{ml})$-stimulated corneal fibroblasts. ChIP assays were performed with H3K27me3 and H3K9me3 antibodies. (mean $\pm S E ;{ }^{* *} P<0.01$ vs. control, $n=3$ ). Figure S3. SET7/9 knockdown attenuated the TGFb1-induced expression of TGFBIp and ECM-associated genes. Wild-type and GCD2-homozygous corneal fibroblasts were infected with SET7/9 or control shRNA lentivirus. After puromycin selection, SET7/9, H3K4me1, TGFBlp, and actin protein levels were analyzed by western blot (A) and infected cells were stimulated with TGFb1 (5 ng/ml) for $8 \mathrm{~h}$, and mRNA and protein levels of TGFBlp were analyzed by RT-qPCR (B) and western blot (C). mRNA levels of ECM associated genes were analyzed by RT-qPCR (D). (mean \pm standard error (SE); ${ }^{* *} P<0.01$ vs. control, $n=3$ ). Figure S4. SET7/9 knockdown attenuated TGFb1-induced increases in H3K4me1 levels at the promoters of TGFBIp and ECM-associated genes. SET7/9 or control shRNA lentivirus infected cells were stimulated with TGFb1 (5 ng/ml) for $8 \mathrm{~h}$, and H3K4me1 levels at the indicated gene promoters were analyzed. ChIP assays were performed with $\mathrm{H} 3 \mathrm{~K} 4 \mathrm{me} 1$ antibody. (mean $\pm \mathrm{SE}$ ); ${ }^{* *} \mathrm{P}<0.01$ vs. control, $n=3$ ).

Competing interests

The authors declared that they have no competing interest.

\section{Authors' contributions}

Conceived and designed the experiments: YSM, GHL. Performed the experiments: YSM, GHL. Analyzed the data: YSM, SIC, EKK. Contributed reagents/materials/analysis tools: YSM, SIC, EKK. Wrote the manuscript: YSM, KSK, EKK. All authors read and approved the final manuscript.

\section{Acknowledgments}

This work was supported by the National Research Foundation of Korea (NRF) grant funded by the Korea government (MEST) (No. 2011-0028699). This research was supported by a Basic Science Research Program through the National Research Foundation of Korea (NRF) funded by the Ministry of Education (NRF 2014R1A1A2057458)

\section{Author details}

1Department of Ophthalmology, Corneal Dystrophy Research Institute, Yonsei University College of Medicine, 250 Seongsanno, Seodaemun-gu, Seoul 120-752, South Korea. ${ }^{2}$ Emory University School of Medicine, Atlanta, GA, USA. ${ }^{3}$ Institute of Vision Research, Severance Biomedical Science Institute, Brain Korea 21 Plus Project for Medical Science, Yonsei University College of Medicine, Seoul, South Korea.

Received: 21 April 2015 Accepted: 4 November 2015 Published online: 09 November 2015

\section{References}

1. Munier FL, Korvatska E, Djemai A, Le Paslier D, Zografos L, Pescia G, et al. Kerato-epithelin mutations in four 5q31-linked corneal dystrophies. Nat Genet. 1997;15:247-51.

2. Korvatska E, Henry H, Mashima Y, Yamada M, Bachmann C, Munier FL, et al. Amyloid and non-amyloid forms of 5q31-linked corneal dystrophy resulting from kerato-epithelin mutations at Arg-124 are associated with abnormal turnover of the protein. J Biol Chem. 2000;275:11465-9.

3. Skonier J, Neubauer M, Madisen L, Bennett K, Plowman GD, Purchio AF. cDNA cloning and sequence analysis of beta ig-h3, a novel gene induced in a human adenocarcinoma cell line after treatment with transforming growth factor-beta. DNA Cell Biol. 1992;11:511-22.

4. Billings PC, Herrick DJ, Kucich U, Engelsberg BN, Abrams WR, Macarak EJ, et al. Extracellular matrix and nuclear localization of beta ig-h3 in human bladder smooth muscle and fibroblast cells. J Cell Biochem. 2000;79:261-73. 
5. Andersen RB, Karring H, Moller-Pedersen T, Valnickova Z, Thogersen IB, Hedegaard CJ, et al. Purification and structural characterization of transforming growth factor beta induced protein (TGFBIp) from porcine and human corneas. Biochemistry. 2004;43:16374-84.

6. LeBaron RG, Bezverkov KI, Zimber MP, Pavelec R, Skonier J, Purchio AF. Beta $\mathrm{IG}-\mathrm{H} 3$, a novel secretory protein inducible by transforming growth factorbeta, is present in normal skin and promotes the adhesion and spreading of dermal fibroblasts in vitro. J Invest Dermatol. 1995;104:844-9.

7. Kim JE, Jeong HW, Nam JO, Lee BH, Choi JY, Park RW, et al. Identification of motifs in the fasciclin domains of the transforming growth factor-betainduced matrix protein betaig-h3 that interact with the alphavbeta5 integrin. J Biol Chem. 2002;277:46159-65.

8. Park SW, Bae JS, Kim KS, Park SH, Lee BH, Choi JY, et al. Beta ig-h3 promotes renal proximal tubular epithelial cell adhesion, migration and proliferation through the interaction with alpha3beta1 integrin. Exp Mol Med. 2004;36:211-9.

9. Klintworth GK. Advances in the molecular genetics of corneal dystrophies. Am J Ophthalmol. 1999;128:747-54.

10. Okada M, Yamamoto S, Inoue $Y$, Watanabe H, Maeda N, Shimomura Y, et al. Severe corneal dystrophy phenotype caused by homozygous R124H keratoepithelin mutations. Invest Ophthalmol Vis Sci. 1998;39:1947-53.

11. Runager K, Basaiawmoit RV, Deva T, Andreasen M, Valnickova Z, Sorensen CS, et al. Human phenotypically distinct TGFBI corneal dystrophies are linked to the stability of the fourth FAS1 domain of TGFBIp. J Biol Chem. 2011;286:4951-8

12. Yellore VS, Rayner SA, Aldave AJ. TGFB1-induced extracellular expression of TGFBIp and inhibition of TGFBIp expression by RNA interference in a human corneal epithelial cell line. Invest Ophthalmol Vis Sci. 2011;52:757-63.

13. Jeon ES, Kim JH, Ryu H, Kim EK. Lysophosphatidic acid activates TGFBIp expression in human corneal fibroblasts through a TGF-beta1-dependent pathway. Cell Signal. 2012;24:1241-50.

14. Yam GH, Wang K, Jhanji V, Choy KW, Baum L, Pang CP. In vitro amyloid aggregate forming ability of TGFBI mutants that cause corneal dystrophies. Invest Ophthalmol Vis Sci. 2012;53:5890-8.

15. Sakharova OV, Taal MW, Brenner BM. Pathogenesis of diabetic nephropathy: focus on transforming growth factor-beta and connective tissue growth factor. Curr Opin Nephrol Hypertens. 2001;10:727-38.

16. Guha M, Xu ZG, Tung D, Lanting L, Natarajan R. Specific down-regulation of connective tissue growth factor attenuates progression of nephropathy in mouse models of type 1 and type 2 diabetes. FASEB J. 2007;21:3355-68.

17. Baricos WH, Reed JC, Cortez SL. Extracellular matrix degradation by cultured mesangial cells: mediators and modulators. Exp Biol Med (Maywood). 2003;228:1018-22

18. Shi $Y$, Massague J. Mechanisms of TGF-beta signaling from cell membrane to the nucleus. Cell. 2003;113:685-700.

19. Hua X, Liu X, Ansari DO, Lodish HF. Synergistic cooperation of TFE3 and smad proteins in TGF-beta-induced transcription of the plasminogen activator inhibitor-1 gene. Genes Dev. 1998;12:3084-95.

20. Kato M, Zhang J, Wang M, Lanting L, Yuan H, Rossi JJ, et al. MicroRNA-192 in diabetic kidney glomeruli and its function in TGF-beta-induced collagen expression via inhibition of E-box repressors. Proc Natl Acad Sci U S A. 2007;104:3432-7.

21. Kato M, Putta S, Wang M, Yuan H, Lanting L, Nair I, et al. TGF-beta activates Akt kinase through a microRNA-dependent amplifying circuit targeting PTEN. Nat Cell Biol. 2009;11:881-9.

22. Schnaper HW, Hayashida T, Hubchak SC, Poncelet AC. TGF-beta signal transduction and mesangial cell fibrogenesis. Am J Physiol Renal Physiol. 2003;284:F243-52.

23. Berger SL. Histone modifications in transcriptional regulation. Curr Opin Genet Dev. 2002;12:142-8.

24. Shilatifard A. Chromatin modifications by methylation and ubiquitination: implications in the regulation of gene expression. Annu Rev Biochem. 2006;75:243-69.

25. Jenuwein T, Allis CD. Translating the histone code. Science. 2001;293:1074-80.

26. Martin C, Zhang Y. The diverse functions of histone lysine methylation. Nat Rev Mol Cell Biol. 2005;6:838-49.

27. Ruthenburg AJ, Allis CD, Wysocka J. Methylation of lysine 4 on histone $\mathrm{H3}$ : intricacy of writing and reading a single epigenetic mark. Mol Cell. 2007;25:15-30

28. Hampsey M, Reinberg D. Tails of intrigue: phosphorylation of RNA polymerase II mediates histone methylation. Cell. 2003;113:429-32.
29. Shi Y, Whetstine JR. Dynamic regulation of histone lysine methylation by demethylases. Mol Cell. 2007;25:1-14.

30. Choi SI, Kim TI, Kim KS, Kim BY, Ahn SY, Cho HJ, et al. Decreased catalase expression and increased susceptibility to oxidative stress in primary cultured corneal fibroblasts from patients with granular corneal dystrophy type II. Am J Pathol. 2009;175:248-61.

31. Pomraning KR, Smith KM, Freitag M. Genome-wide high throughput analysis of DNA methylation in eukaryotes. Methods. 2009;47:142-50.

32. Villeneuve LM, Reddy MA, Lanting LL, Wang M, Meng L, Natarajan R. Epigenetic histone $\mathrm{H} 3$ lysine 9 methylation in metabolic memory and inflammatory phenotype of vascular smooth muscle cells in diabetes. Proc Natl Acad Sci U S A. 2008;105:9047-52.

33. Choi SI, Kim BY, Dadakhujaev S, Oh JY, Kim TI, Kim JY, et al. Impaired autophagy and delayed autophagic clearance of transforming growth factor beta-induced protein (TGFBI) in granular corneal dystrophy type 2 . Autophagy. 2012;8:1782-97.

34. Wang X, Zhu K, Li S, Liao Y, Du R, Zhang X, et al. MLL1, a H3K4 methyltransferase, regulates the TNFalpha-stimulated activation of genes downstream of NF-kappaB. J Cell Sci. 2012;125:4058-66.

35. Mishra BP, Ansari KI, Mandal SS. Dynamic association of MLL1, H3K4 trimethylation with chromatin and Hox gene expression during the cell cycle. FEBS J. 2009;276:1629-40.

36. Guenther MG, Jenner RG, Chevalier B, Nakamura T, Croce CM, Canaani E, et al. Global and Hox-specific roles for the MLL1 methyltransferase. Proc Natl Acad Sci U S A. 2005;102:8603-8.

37. Fischle W, Wang Y, Jacobs SA, Kim Y, Allis CD, Khorasanizadeh S. Molecular basis for the discrimination of repressive methyl-lysine marks in histone $\mathrm{H} 3$ by Polycomb and HP1 chromodomains. Genes Dev. 2003;17:1870-81.

38. Bannister AJ, Kouzarides T. Regulation of chromatin by histone modifications. Cell Res. 2011;21:381-95.

39. Wang H, Cao R, Xia L, Erdjument-Bromage H, Borchers C, Tempst P, et al. Purification and functional characterization of a histone $\mathrm{H} 3$-lysine 4-specific methyltransferase. Mol Cell. 2001;8:1207-17.

40. Nishioka K, Chuikov S, Sarma K, Erdjument-Bromage H, Allis CD, Tempst P, et al. Set9, a novel histone $\mathrm{H} 3$ methyltransferase that facilitates transcription by precluding histone tail modifications required for heterochromatin formation. Genes Dev. 2002;16:479-89.

41. Chuikov S, Kurash JK, Wilson JR, Xiao B, Justin N, Ivanov GS, et al. Regulation of p53 activity through lysine methylation. Nature. 2004;432:353-60.

42. Berger SL. The complex language of chromatin regulation during transcription. Nature. 2007:447:407-12.

43. Esteve PO, Chin HG, Benner J, Feehery GR, Samaranayake M, Horwitz GA et al. Regulation of DNMT1 stability through SET7-mediated lysine methylation in mammalian cells. Proc Natl Acad Sci U S A. 2009;106:5076-81.

44. Kouskouti A, Scheer E, Staub A, Tora L, Talianidis I. Gene-specific modulation of TAF10 function by SET9-mediated methylation. Mol Cell. 2004;14:175-82.

45. Ea CK, Baltimore D. Regulation of NF-kappaB activity through lysine monomethylation of p65. Proc Natl Acad Sci U S A. 2009;106:18972-7.

46. Yang XD, Huang B, Li M, Lamb A, Kelleher NL, Chen LF. Negative regulation of NF-kappaB action by Set9-mediated lysine methylation of the RelA subunit. EMBO J. 2009;28:1055-66.

47. Li Y, Reddy MA, Miao F, Shanmugam N, Yee JK, Hawkins D, et al. Role of the histone $\mathrm{H} 3$ lysine 4 methyltransferase, SET7/9, in the regulation of NF-kappaB-dependent inflammatory genes. Relevance to diabetes and inflammation. J Biol Chem. 2008;283:26771-81.

48. El-Osta A, Brasacchio D, Yao D, Pocai A, Jones PL, Roeder RG, et al. Transient high glucose causes persistent epigenetic changes and altered gene expression during subsequent normoglycemia. J Exp Med. 2008;205:2409-17.

49. Pons D, de Vries FR, van den Elsen PJ, Heijmans BT, Quax PH, Jukema JW. Epigenetic histone acetylation modifiers in vascular remodelling: new targets for therapy in cardiovascular disease. Eur Heart J. 2009;30:266-77. 